\title{
ON THE POSITIVE SOLUTIONS OF A HIGHER ORDER FUNCTIONAL DIFFERENTIAL EQUATION WITH A DISCONTINUITY
}

\author{
JOHN R. GRAEF, PAUL W. SPIKES \\ and MYRON K. GRAMMATIKOPOULOS \\ Department of Mathematics \\ Mississippi State University \\ Mississippi State, Mississippi 39762 U.S.A. \\ (Received August 7, 1981)
}

ABSTRACT. The $n$-th order nonlinear functional differential equation

$$
\left[r(t) x^{(n-v)}(t)\right]^{(v)}=f(t, x(g(t)))
$$

is considered; necessary and sufficient conditions are given for this equation to have: (i) a positive bounded solution $x(t) \rightarrow B>0$ as $t \rightarrow \infty$; and (ii) all positive bounded solutions converging to 0 as $t \rightarrow \infty$. Other results on the asymptotic behavior of solutions are also given. The conditions imposed are such that the equation with a discontinuity

$$
\left[r(t) x^{(n-\nu)}(t)\right]^{(\nu)}=q(t) x^{-\lambda}, \lambda>0
$$

is included as a special case.

KEY WORDS AND PHRASES. Functional differential equations, discontinuous right hand side, positive solutions, asymptotic behavior.

\section{MATHEMATICS SUBJECT CLASSIFICATION CODES. Primary: $34 \mathrm{~K} 15,34 \mathrm{C} 11$.}

\section{INTRODUCTION.}

Kitamura and Kusano [1] have recently studied the problems of the existence and asymptotic behavior of positive solutions of the equation

$$
\left[r(t) x^{\prime}\right]^{\prime}=q(t) x^{-\lambda}
$$

where $r$ and $q$ are positive and continuous on $[0, \infty)$ and $\lambda$ is a positive constant. Other authors, including Taliaferro [2] and [3], have studied the behavior of the solutions of (1.1) with either $q(t)<0$ or $\lambda<0$. However, as pointed out in [1], 
there seems to be no literature concerning (1.1) with $q(t)>0$ and $\lambda>0$ other than [1] and a superficial treatment of some of its special cases by Kamke[4]. Here we are concerned with the existence and the asymptotic properties of the positive continuable solutions of the functional differential equation

$$
\left[r(t) x^{(n-v)}(t)\right]^{(v)}=f(t, x(g(t)))
$$

where $1 \leq v \leq n-1, r, g:\left[t_{0}, \infty\right) \rightarrow R$ are continuous, $r(t)>0, \int^{\infty}[1 / r(s)] d s=\infty$, $g(t) \rightarrow \infty$ as $t \rightarrow \infty$ and $f(t, y)$ is positive, continuous, and nonincreasing with respect to $\mathrm{y}$ on $\left[t_{0}, \infty\right) \mathrm{x}(0, \infty)$. Clearly $(1.1)$ is a special case of $(1.2)$ and the results here extend some of those obtained in [1].

\section{EXISTENCE AND ASYMPTOTIC PROPERTIES.}

We first state two lemmas which will be used in some of our proofs.

LEMMA 1. ([5,6; Lemma 1$])$. Let $u$ be a positive (n- $v)$-times continuously differentiable function on the interval $[a, \infty)$ and let $\mu$ be a positive continuous function on $[a, \infty)$ such that

and the function $w \equiv \mu u^{(n-\nu)}$ is $\nu$-times continuously differentiable on $[a, \infty)$. Moreover, let

$$
w_{k}= \begin{cases}u^{(k)}, & \text { if } 0 \leq k \leq n-v-1 \\ w^{(k-n+v)}, & \text { if } n-v \leq k \leq n .\end{cases}
$$

If $\omega_{n}(t) \equiv w^{(\nu)}(t)$ is of constant sign and not identically zero for all large $t$, then there exists $t_{\mathrm{u}} \geq \mathrm{a}$ and an integer $l, 0 \leq \ell \leq \mathrm{n}$, with $\mathrm{n}+\ell$ even for $\omega_{\mathrm{n}}$ nonnegative or $n+l$ odd for $\omega_{n}$ non positive, and such that for every $t \geq t_{u}$

$$
\ell>0 \text { implies } \omega_{k}(t)>0(k=0,1, \ldots, \ell-1)
$$

and

$$
\ell \leq n-1 \text { imp1ies }(-1)^{\ell+k} \omega_{k}(t)>0(k=\ell, \ell+1, \ldots, n-1) .
$$

LEMIA 2. ([5,6; Lemma 2]). If the functions $u, \mu, w$ and $\omega_{k}$ are as in Lemma 1 and for some $k=0,1, \ldots, n-2 \omega_{k}(t) \rightarrow c$ as $t \rightarrow \infty$, then $w_{k+1}(t) \rightarrow 0$ as $t \rightarrow \infty$.

It will be convenient to make use of the following notation in the remainder of this paper. For any $\mathrm{T} \geq \mathrm{t}_{0}$ and $\mathrm{a} 11 \mathrm{t} \geq \mathrm{T}$ we let 


$$
\begin{aligned}
& z(t)=r(t) x^{(n-v)}(t), \\
& \omega_{k}(t)=\left\{\begin{array}{l}
x^{(k)}(t), \quad 0 \leq k \leq n-v-1 \\
z^{(k-n+\nu)}(t), n-\nu \leq k \leq n,
\end{array}\right. \\
& J(T, t)=\int_{T}^{t}\left[(t-s)^{n-v-1} s^{\nu-1} / r(s)\right] d s /(n-v-1) !(\nu-1) !,
\end{aligned}
$$

and

$$
S(T, t)=\int_{T}^{t}\left[(t-s)^{\nu-1} s^{n-v-1} / r(s)\right] d s /(n-v-1) !(\nu-1) !
$$

From our assumptions regarding the functions in (1.2), it is easy to see that if $x(t)$ is a positive continuable solution of (1.2), then there exists $t \geq t_{0}$ such that $x(t)$ belongs to one of the two classes:

$$
\text { (I) } \omega_{n-1}(t)=z^{(\nu-1)}(t)>0 \text { for } t \geq t_{1}
$$

or

$$
\text { (II) } w_{n-1}(t)=z^{(\nu-1)}(t)<0 \text { for } t \geq t_{1} \text {. }
$$

As is indicated in the discussion following its proof, our first theorem is very near being a necessary and sufficient result.

THEOREM 1. Let $x(t)$ be a positive solution of type (I).

(i) If for every constant $c>0$

$$
\int^{\infty} f\left(s, c J\left(t_{0}, g(s)\right) d s<\infty,\right.
$$

then there exists a positive constant A such that

$$
[x(t) / J(T, t)] \rightarrow A \text { as } t \rightarrow \infty
$$

for all sufficiently large $T$.

(ii) If (2.2) holds then (2.1) holds for some constant $c>0$.

PROOF. let $x(t)$ be a positive solution of (1.2) of type (I), then there exists $t_{2}>\max \left\{t_{1}, 0\right\}$ such that $x(t), x(g(t))$, and $z^{(\nu-1)}(t)$ are all positive on $\left[t_{2}, \infty\right)$. Since $x(t)>0$ implies that $z^{(\nu)}(t)>0$, we see that $z^{(\nu-1)}(t)$ is increasing on $\left[t_{2}, \infty\right)$. It then follows from Lemma 2 that $\omega_{k}(t) \rightarrow \infty$ as $t \rightarrow \infty$ for $k=0,1, \ldots, n-2$. Then, by repeated application of L'Hôpital's rule, we obtain

$$
\lim _{t \rightarrow \infty}\left[x(t) / J\left(t_{2}, t\right)\right]=\lim _{t \rightarrow \infty} z^{(\nu-1)}(t) /(\nu-1) !>K
$$


for some positive constant $K$. Thus there exists $T \geq t_{2}$ so that

$$
x(g(t)) \geq K J(T, g(t))
$$

for $t \geq T$. To show that (2.2) holds it suffices (in view of (2.3)) to show that $x(t) / J(T, t)$ is bounded above. For this purpose we integrate (1.2) n-times over $[\mathrm{T}, \mathrm{t}]$ obtaining

$$
\begin{aligned}
x(t)= & Q_{n-v-1}(t)+\int_{T}^{t}\left[(t-s)^{n-v-1} / r(s)\right] P_{\nu-1}(s) d s /(n-v-1) ! \\
& +\int_{T}^{t}\left[(t-s)^{n-v-1} / r(s)\right] \int_{T}^{s}(s-u)^{\nu-1} f(u, x(g(u))) \text { duds/(n-v-1)!(v-1)!, }
\end{aligned}
$$

where $Q_{n-V-1}(t)$ and $P_{\nu-1}(t)$ are polynomials of degree at most $n-v-1$ and $\nu-1$ respectively. By (2.4), we see that

$$
\int_{T}^{s}(s-u)^{\nu-1} f\left(u, x(g(u)) d u \leq s^{\nu-1} \int_{T}^{t} f(u, K J(T, g(u))) d u,\right.
$$

and therefore we have

$$
x(t) / J(T, t) \leq \int_{T}^{\infty} f(x, K J(T, g(s))) d s+H
$$

for some positive constant H. (2.1) implies that $x(t) / J(T, t)$ is bounded above.

Now suppose that (2.2) holds; then there exists positive constants $A_{1}$ and $T_{1} \geq T$ such that $x(t) \leq A_{1} J\left(T_{1}, t\right)$ and $x(g(t)) \leq A_{1} J\left(T_{1}, g(t)\right)$ for $t \geq T_{1}$. But (2.5) holds with $\mathrm{T}$ replaced by $\mathrm{T}_{1}$ so we have

$$
\begin{aligned}
& A_{1} \geq x(t) / J\left(T_{1}, t\right) \\
& \quad \geq\left[1 / J\left(T_{1}, t\right)\right]\left\{Q_{n-v-1}(t)+\int_{T_{1}}^{t}\left[(t-s)^{n-v-1} / r(s)\right] P_{v-1}(s) d s /(n-v-1) !\right. \\
& \left.\quad+\int_{T_{1}}^{t}\left[(t-s)^{n-v-1} / r(s)\right] \int_{T_{1}}^{s}(s-u)^{v-1} f\left(u, A_{1} J\left(T_{1}, g(u)\right)\right) d u d s /(n-v-1) !(v-1) !\right\}
\end{aligned}
$$

Therefore

$$
A_{1} \geq \lim _{t \rightarrow \infty}\left[x(t) / J\left(T_{1}, t\right)\right] \geq \lim _{t \rightarrow \infty} \int_{T_{1}}^{t} f\left(s, A_{1} J\left(T_{1}, g(s)\right)\right) d s
$$

which implies that

$$
\int_{T_{1}}^{\infty} f\left(s, A_{1} J\left(T_{1}, g(s)\right)\right) d s<\infty,
$$

and it is not difficult to see that this implies that (2.1) holds for some positive constant $\mathrm{c}$. 
Notice that if the function $f$ were such that condition (2.1) holding for some c $>0$ implies that (2.1) holds for all positive constants, then condition (2.1) would be necessary and sufficient for every positive solution of (1.2) of type (I) to satisfy (2.2). This would be the case if $f(t, y)$ were homogeneous of some degree $\alpha$ in $y$, i.e. $f(t, s y)=s^{\alpha} f(t, y)$. Since $f(t, y)=q(t) y^{-\lambda}, \lambda>0$, is homogeneous of degree $s=-\lambda$, we see that our Theorem 1 includes Theorem 1 and part (ii) of Theorem 4 in [1]. A simple example to which Theorem 1 applies is the equation

$$
\left[x^{\prime}(t) / t\right]^{\prime \prime}=6\left(\ln t+t^{3}\right)^{2} / t^{4} x^{2}(t), \quad t>1
$$

which has the positive solution $x(t)=1 n t+t^{3}$. Notice that here $J(T, t)=$ $\left(t^{3}-T^{3}\right) / 3$ so that

$$
A=\lim _{t \rightarrow \infty}[x(t) / J(T, t)]=\lim _{t \rightarrow \infty} 3\left(\ln t+t^{3}\right) /\left(t^{3}-T^{3}\right)=3 .
$$

THEOREM 2. A necessary and sufficient condition for (1.2) to have a bounded positive solution $x(t)$ satisfying $x(t) \rightarrow B>0$ as $t \rightarrow \infty$ is that $\int^{\infty} s(T, s) f(s, c) d s<\infty$ for some constant $c>0$ and all sufficiently large $\mathrm{T}$.

PROOF. To prove necessity let $x(t)$ be a positive bounded solution of (1.2) and let $c>0$ and $T>\max \left\{t_{0}, 0\right\}$ be such that

$$
0<x(g(t)) \leq c, \quad t \geq T .
$$

Notice that from $(1.2)$ we have $z^{(\nu)}(t)>0$ for $t \geq T$ so that the hypotheses of Lemma 1 are satisfied. Also, it is easy to see that the boundedness of $x(t)$ implies that the integer $\ell$ assigned to $x(t)$ by Lemma 1 must satisfy $\ell<2$. Moreover, since $z^{(v)}(t)>0$ implies that $n+l$ is even, we see that $l=0$ for $n$ even and $l=1$ for $\mathrm{n}$ odd. Consequently, by Lemma 1 , we have for $\mathrm{n}$ even that

$$
(-1)^{i} w_{i}(t)>0, \quad i=1,2, \ldots, n-1
$$

and for $n$ odd that

$$
(-1)^{i+1} \omega_{i}(t)>0, \quad i=1,2, \ldots, n-1
$$

for $t \geq T$. Next we multiply $(1.2)$ by $S(T, t)$ and integrate to obtain

$$
\int_{T}^{t} S(T, s) f(s, x(g(s))) d s=\int_{T}^{t} S(T, s) z^{(\nu)}(s) d s .
$$

But successive integration by parts yields 


$$
\begin{aligned}
\int_{T}^{t} S^{\prime}(T, S) z^{(\nu)}(s) d s= & S(T, t) z^{(\nu-1)}(t)-S^{\prime}(T, t) z^{(\nu-2)}(t)+\ldots+(-1)^{\nu-1} S^{(\nu-1)}(t) z(t) \\
& +(-1)^{\nu} t^{n-\nu-1} x^{(n-\nu-1)}(t) /(n-\nu-1) !+\ldots+(-1)^{n-1} x(t)+L
\end{aligned}
$$

where $L$ is a constant. Therefore, in view of (2.7) and (2.8), it follows from (2.9) that $\int_{T}^{\infty} S(T, s) f(s, x(g(s))) d s<\infty$ and by $(2.6)$ we see that

$$
\int_{\mathrm{T}}^{\infty} \mathrm{S}(\mathrm{T}, \mathrm{s}) \mathrm{f}(\mathrm{s}, \mathrm{c}) \mathrm{ds}<\infty \text {. }
$$

To prove sufficiency, let $\mathrm{T}_{0}>\max \left\{\mathrm{t}_{0}, 0\right\}$ and $c>0$ be such that

$$
\int_{\mathrm{T}}^{\infty} \mathrm{S}\left(\mathrm{T}_{0}, \mathrm{~s}\right) \mathrm{f}(\mathrm{s}, \mathrm{c}) \mathrm{ds}<\mathrm{c}
$$

and consider the integral equation

$$
x(t)=2 c+(-1)^{n} \int_{t}^{\infty}\left(\int_{t}^{s}\left[(s-u)^{\nu-1}(u-t)^{n-v-1} / r(u)\right] d u\right) f(s, x(g(s))) d s /(v-1) !(n-v-1) ! \text {. }
$$

It is not difficult to verify by differentiation that a solution of (2.11) is also a solution of (1.2). We will show that (1.2) has a solution $x(t) \rightarrow B>0$ as $t \rightarrow \infty$ by using the following special case of Tychonov's fixed point theorem:

THEOREM. Let $\mathrm{F}$ be a Fréchet space and $\mathrm{X}$ be a closed convex subset of $\mathrm{F}$. If $\mathrm{G}: \mathrm{X} \rightarrow \mathrm{X}$ is continuous and the closure $\overline{\mathrm{G}(\mathrm{X})}$ is a compact subset of $\mathrm{X}$, then there exists at least one fixed point $x$ in $X$.

In order to utilize this theorem, let $u_{0}=\min \left\{T_{0}, \min _{t \geq t_{0}} g(t)\right\}$ and let $F$ be the Fréchet space of all continuous functions $x:\left[u_{0}, \infty\right) \rightarrow R$ with the topology of uniform convergence on compact subintervals of $\left[u_{0}, \infty\right)$. Let the closed convex subset $X$ of $F$ defined by

$$
X=\left\{x \in F: c \leq x(g(t)) \leq 3 c, t \geq u_{0}\right\},
$$

and define the operator $G$ on $X$ by

where

$$
(G x)(t)=\left\{\begin{array}{l}
2 c+(-1)^{n} Q(t), \text { if } t \geq T_{0} \\
2 c+(-1)^{n} Q\left(T_{0}\right), \text { if } u_{0} \leq t \leq T_{0}
\end{array}\right.
$$

$$
Q(m)=\int_{m}^{\infty}\left(\int_{m}^{s}\left[(s-u)^{v-1}(u-m)^{n-v-1} / r(u)\right] d u\right) f(s, x(g(s))) d s /(n-v-1) !(v-1) ! .
$$


To complete the proof we show that G satisfies all the hypotheses of the fixed point theorem stated above. First observe that for any $x \in X$

$$
|(G x)(t)-2 c| \leq Q\left(T_{0}\right)
$$

for $t \geq u_{0}$, and that

$$
Q\left(T_{0}\right) \leq \int_{T_{0}}^{\infty} S\left(T_{0}, s\right) f(s, c) d s<c .
$$

Thus we see that $G$ maps $X$ into $X$.

To show that $G$ is continuous let $\left\{x_{\lambda}\right\}, \lambda=1,2, \ldots$ be any sequence of functions in $\mathrm{X}$ converging uniformly to $\mathrm{x} \varepsilon \mathrm{X}$ on every compact subinterval of $\left[u_{0}, \infty\right)$. Let $t \geq u_{0}$ and $T_{2}>\max \left\{t, T_{0}\right\}$, then $f(t, x(g(t))) \rightarrow f(t, x(g(t)))$ uniformly on $\left[u_{0}, T_{2}\right]$. However,

$$
\left|\left(G x_{\lambda}\right)(t)-(G x)(t)\right| \leq \int_{T_{0}}^{T_{2}} s\left(T_{0}, s\right)\left|f\left(s, x_{\lambda}(g(s))\right)-f(s, x(g(s)))\right| d s,
$$

and we see from (2.10) that $\mathrm{Gx}_{\lambda}$ converges uniformly to $G \mathrm{x}$ on any compact subinterval of $\left[u_{0}, \infty\right)$. Hence, we conclude that $G$ is continuous.

Finally, in order to show that $\overline{G X}$ is a compact subset of $X$, it is sufficient to show that $\mathrm{GX}$ is relatively compact since $\mathrm{GX} \subset \mathrm{X}$ and $\mathrm{X}$ is closed. Furthermore, since $X$ is bounded, it suffices to show that GX is equicontinuous. For this purpose, we distinguish two cases. If $n-v \neq 1$, then from the definitions of GX, $S$, and $x$, we have that there exists a constant $L_{1}$ such that

$$
\begin{aligned}
\left|(G x)^{\prime}(t)\right| & \leq \int_{T}^{\infty}\left(\int_{t}^{s}\left[(s-u)^{v-1}(u-t)^{n-v-2} / r(u)\right] d u\right) f(s, x(g(s))) d s /(v-1) !(n-v-2) ! \\
& \leq L_{1} \int_{T}^{\infty} S\left(T_{0}, s\right) f(s, c) d s
\end{aligned}
$$

for $t \geq u_{0}$. Hence it follows from (2.10) that there is constant $\mathrm{L}_{2}$ such that $\left|(G x)^{\prime}(t)\right| \geq L_{2}$ where $L_{2}$ is independent of both $x$ and $t$. It then follows that GX is equicontinuous on $\left[u_{0}, \infty\right)$.

If $n-v=1$, we have for each $t \geq T_{0}$ that

$$
\left|(G x)^{\prime}(t)\right| \leq L_{3} \int_{t}^{\infty}(s-t)^{\nu-1} f(s, x(g(s))) d s / r(t)
$$

for some positive constant $L_{3}$. Since $G x$ is constant on $\left[u_{0}, T_{0}\right]$, then 


$$
\left|(G x)^{\prime}(t)\right| \leq L_{3} \int_{T_{0}}^{\infty}\left(s-T_{0}\right)^{\nu-1} f(s, c) d s / r(t)
$$

for all $t \geq u_{0}$. Noticing that $\left[\left(s-T_{0}\right)^{\nu-1} / s\left(T_{0}, s\right)\right] \rightarrow 0$ as $s \rightarrow \infty$, we see that

$$
\left|(G x)^{\prime}(t)\right| \leq\left[L_{4} / r(t)\right] \int_{T_{0}}^{\infty} S\left(T_{0}, s\right) f(s, c) d s
$$

for some constant $\mathrm{L}_{4}$. Therefore, for any given closed subinterval $\left[\mathrm{u}_{0}, \mathrm{~T}_{1}\right]$, of $\left[u_{0}, \infty\right)$, with $T_{1}>T_{0}$, there exists a constant $L\left(T_{1}\right)$ such that

$$
\left|(G x)^{\prime}(t)\right| \leq L\left(T_{1}\right)
$$

where $L\left(T_{1}\right)$ is independent of both $x \in X$ and $t$ in $\left[u_{0}, T_{1}\right]$. Also, if $t_{2}>t_{1} \geq T_{1}$, then

$$
\begin{aligned}
\left|(G x)\left(t_{2}\right)-(G x)\left(t_{1}\right)\right| & \leq \int_{t_{2}}^{\infty} S\left(T_{1}, s\right) f(x, c) d s+\int_{t_{1}}^{\infty} S\left(T_{1}, s\right) f(s, c) d s \\
& \leq 2 \int_{T_{1}}^{\infty} S\left(T_{1}, s\right) f(s, c) d s,
\end{aligned}
$$

where the last integral tends to zero as $T_{1} \rightarrow \infty$ independent of $x \varepsilon x$ and $t_{1}, t_{2}$ in $\left[\mathrm{T}_{1}, \infty\right)$. If is now easy to see that $\mathrm{GX}$ is equicontinuous on $\left[u_{0}, \infty\right)$ for $\mathrm{n}-\nu=1$.

We now have all the hypotheses of the fixed point theorem satisfied and therefore we have the existence of $\mathrm{x} \varepsilon \mathrm{X}$ such that $\mathrm{Gx}=\mathrm{x}$, i.e. $\mathrm{x}$ is a solution of both (1.2) and (2.11) and satisfies $c \leq x(t) \leq 3 c$. By differentiating both sides of (2.11) we see that $x^{\prime}(t)$ has fixed sign and hence $x(t) \rightarrow B$ as $t \rightarrow \infty$ for some $B$ in $[c, 3 c]$.

REMARK. Theorem 2 reduces to Theorem 2 in [1] when $r(t) \equiv 1, f(t, y)=q(t) y^{-\lambda}$, $\lambda>0$, and $n=2$. It also includes part (iii) of Theorem 4 in [1]. The equations

$$
x^{(2 k)}(t)=c e^{-4 t / 3}\left(e^{t}+c\right)^{1 / 3} x^{-1 / 3}(t), \quad t>0,
$$

where $c$ is any positive constant and $k=1,2, \ldots$ are examples of equations satisfying the hypotheses of Theorem 2. Notice that for each $k, x(t)=e^{-t}\left(e^{t}+c\right)$ is a solution of $(2.12)$ satisfying $x(t) \rightarrow 1$ as $t \rightarrow \infty$.

THEOREM 3. A necessary and sufficient condition for every positive bounded solution $x(t)=\omega_{0}(t)$ of $(1.2)$ to be such that $\omega_{k}(t) \rightarrow 0$ monotonically as $t \rightarrow \infty$ for $\mathrm{k}=0,1, \ldots, \mathrm{n}-1$ is that 


$$
\int^{\infty} S(T, s) f(s, c) d s=\infty
$$

for every $c>0$ and all sufficiently large $\mathrm{T}$.

PROOF. It follows from Theorem 2 that every positive bounded solution $x(t)$ of (1.2) satisfies $\omega_{0}(t)=x(t) \rightarrow 0$ as $t \rightarrow \infty$ if and only if (2.13) holds. Since $\omega_{0}(t) \rightarrow 0$ as $t \rightarrow \infty$, then $\omega_{k}(t) \rightarrow 0$ as $t \rightarrow \infty$ for $k=1,2, \ldots, n-1$ by Lemma 2 .

In contrast to Theorem 2 and 3 , the next two theorems give sufficient conditions for the positive solutions of (1.2) to be unbounded. In their proofs we utilize the function

$$
J_{1}(c, T, t)=\int_{T}^{t}\left[c(t-s)^{n-v-1} h(s) / r(s)\right] d s
$$

where $c$ is a positive constant and $h(t)=t^{\nu-2}$ for $\nu-2 \geq 0$ and $h(t)=1$ for $\nu=1$.

THEOREM 4. If for all positive constants $c$ and $L$ and all sufficiently large $T$

$$
\underset{t \rightarrow \infty}{\lim \sup _{t \rightarrow \infty}}\left\{\int_{T}^{t}\left[(t-s)^{n-v-1} / r(s)\right] \int_{T}^{s}(s-u)^{\nu-1} f\left(u, J_{1}(c, T, g(u))\right) d u d s-L J(T, t)\right\}=\infty
$$

then every positive solution of (1.2) of type (II) is unbounded.

PROOF. Let $x(t)$ be a positive solution of $(1.2)$ of type (II). Since $z^{(v-1)}(t)$ is eventually negative, it is easy to see from Taylor's formula that there exists $T_{1}>\max \left\{0, t_{0}\right\}$ and a positive constant $c_{1}$ so that $r(t) x^{(n-\nu)}(t)=z(t) \leq c_{1} h(t)$, $t \geq T_{1}$. Thus $x^{(n-v)}(t) \leq c_{1} h(t) / r(t)$ for $t \geq T_{1}$. Integrating each member of the last inequality $(n-v)$ times we obtain $x(t) \leq J_{1}\left(c_{2}, T_{1}, t\right)$ for some constant $c_{2}>0$. Hence there exists $\mathrm{T} \geq \mathrm{T}_{1}$ such that

$$
x(g(t)) \leq J_{1}\left(c_{2}, T, g(t)\right), \quad t \geq T .
$$

Next we integrate (1.2) n-times obtaining

$$
\begin{aligned}
x(t)= & Q_{n-v-1}(t)+\int_{T}^{t}\left[(t-s)^{n-v-1} / r(s)\right] P_{v-1}(s) d s /(n-v-1) ! \\
& +\int_{T}^{t}\left[(t-s)^{n-v-1} / r(s)\right] \int_{T}^{s}(s-u)^{v-1} f(u, x(g(u)) \text { duds/(n-v-1)!(v-1)! }
\end{aligned}
$$

where $Q_{n-\nu-1}(t)$ and $P_{\nu-1}(t)$ are polynomials of degree at most $n-v-1$ and $v-1$ respectively. But the last equation, together with (2.15), implies that there exists constants $c_{3}>0$ and $L>0$ such that 


$$
c_{3} x(t) \geq-L J(T, t)+\int_{T}^{t}\left[(t-s)^{n-V-1} / r(s)\right] \int_{T}^{s}(s-u)^{\nu-1} f\left(u, J_{1}\left(c_{2}, T, g(u)\right)\right) d u d s,
$$

and the conclusion of the theorem follows form (2.14).

REMARK. It is easy to see from the proof of Theorem 4 that, if (2.14) were replaced by

$$
\underset{t \rightarrow \infty}{\lim \inf }\left\{\int_{T}^{t}\left[(t-s)^{n-v-1} / r(s)\right] \int_{T}^{s}(s-u)^{\nu-1} f\left(u, J_{1}(c, T, g(u))\right) d u d s-L J(T, t)\right\}>0,
$$

in the hypotheses of Theorem 4, then we could conclude that every positive solution of (1.2) of type (II) is bounded away from zero.

THEOREM 5. Suppose that

$$
\int^{\infty} f\left(s, J_{1}(c, T, g(s)) d s=\infty\right.
$$

for every constant $c>0$ and all sufficiently large $T$. If $x(t)$ is a positive solution of $(1.2)$, then $x(t)$ is of type (I) and there exists positive constants $c, t_{2}$ and $T_{1} \geq t_{2}$ such that $x(t) \geq c J\left(t_{2}, t\right)$ for $t \geq T_{1}$.

PROOF. Let $x(t)$ be a positive solution of (1.2) then there exists $t_{2}>\max \left\{t_{0}, 0\right\}$ so that $x(t), x(g(t)), z^{(\nu)}(t)$ and $\left|z^{(\nu-1)}(t)\right|$ are all positive on $\left[t_{2}, \infty\right)$. First suppose that $x(t)$ is of type $(I I)$, i.e. $z^{(V-1)}(t)<0$ for $t \geq t_{2}$. Then, by Taylor's formula, there exists a constant $c_{1}>0$ and $T \geq t_{2}$ such that (2.15) holds for $t \geq T$. From (2.15) and an integration of (1.2) we have

$$
z^{(\nu-1)}(t)=z^{(\nu-1)}(T)+\int_{T}^{t} f(s, x(g(s))) d s \geq z^{(\nu-1)}(T)+\int_{T}^{t} f\left(x, J_{1}(c, T, g(s))\right) d s .
$$

But then (2.16) implies that $z^{(\nu-1)}(t) \rightarrow \infty$ as $t \rightarrow \infty$ contradicting the assumption that $x(t)$ is of type (II). Therefore, we conclude that $x(t)$ is of type (I).

Now from L'Hôpital's rule, we obtain

$$
\lim _{t \rightarrow \infty}\left[x(t) / J\left(t_{2}, t\right)\right]=\lim _{t \rightarrow \infty} z^{(v-1)}(t) /(v-1) !
$$

as in the proof of Theorem 1. Since $x(t)$ being of type (I) implies that $z^{(V-1)}(t)$ is positive and increasing, from (2.17) there exists constants $c>0$ and $T_{1} \geq t_{2}$ such that $x(t) \geq c J\left(t_{2}, t\right)$ for $t \geq T_{1}$.

REMARK. If $\int^{\infty} f\left(s, c J\left(t_{0}, g(s)\right)\right) d s=\infty$ for every constant $c>0$, then if follows from Theorem 1 and condition $(2.17)$ that $\left[x(t) / J\left(t_{2}, t\right)\right] \rightarrow \infty$ as $t \rightarrow \infty$. Notice also 
that $v=1$, then $J_{1}(c, T, t)=c J(T, t)$. If, in addition, $f(t, y)$ is homogeneous of some degree $\alpha$ in $y$, then $\int^{\infty} f\left(s, c J\left(t_{0}, g(s)\right)\right) d s=\infty$ is equivalent to (2.16). Thus Theorem 5 includes the corollary in [1]. The equation

$$
\left(t x^{\prime}(t)\right)^{\prime}=(2+\ln t)(t \ln t)^{2} / x^{2}(t), t>1
$$

satisfies all the hypotheses of Theorem 5 and has the solution $x(t)=t \ln t$. ACKNOWLEDGEMENT. The work of the first and the second authors was supported by the Mississippi State University Biological and Physical Sciences Research Institute. The research of the third author was supported by the Ministry of Coordination of Greece.

\section{REFERENCES}

1. KITAMURA, Y. and KUSANO, T. Positive solutions of a second order differential equation with a discontinuous nonlinear term, (to appear).

2. TALIAFERRO, S. On the positive solutions of $y^{\prime \prime}+\phi(t) y^{-\lambda}=0$, Nonlinear Anal. $\underline{2}(1978), 437-446$.

3. TALIAFERRO, S. Asymptotic behavior of solutions of $y^{\prime \prime}=\phi(t) y^{\lambda}$, J. Math. Anal. App1. 66 (1978), 95-134.

4. KAMKE, E. Differentialgleichungen, Lösungsmethoden und Lösgungen, Vo1. I, Akademische Verlags. Leibzig (1956).

5. GRAMMATIKOPOULOS, M.K. On the effect of deviating arguments on the behavior of bounded solutions of nonlinear differential equations, Ukrain. Mat. $Z$. 30 (1978), 462-473 (Russian).

6. GRAMMATIKOPOULOS, M.K. Asymptotic and oscillation criteria for nonlinear differential equations with deviating arguments, (to appear). 


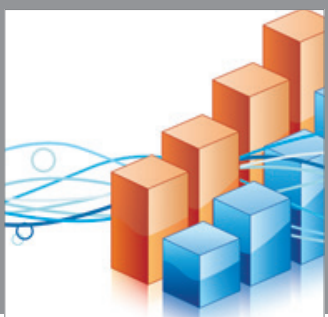

Advances in

Operations Research

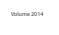

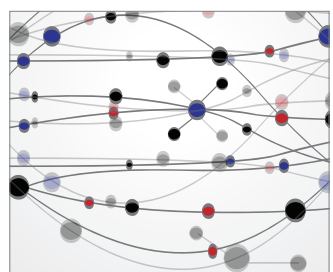

\section{The Scientific} World Journal
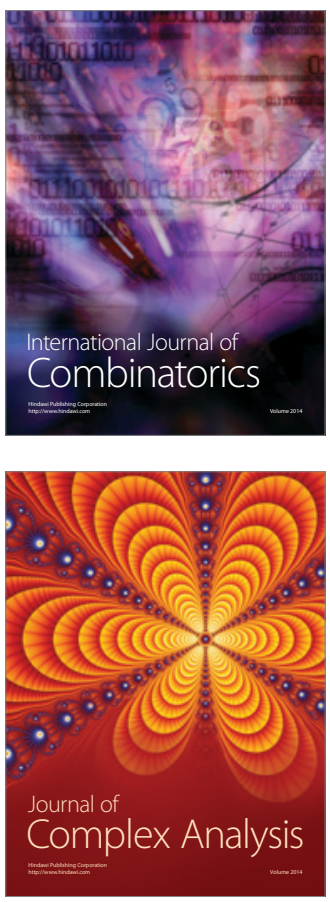

International Journal of

Mathematics and

Mathematical

Sciences
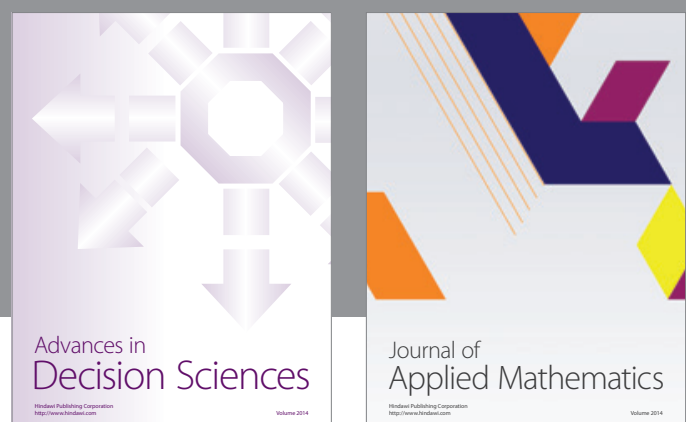

Journal of

Applied Mathematics
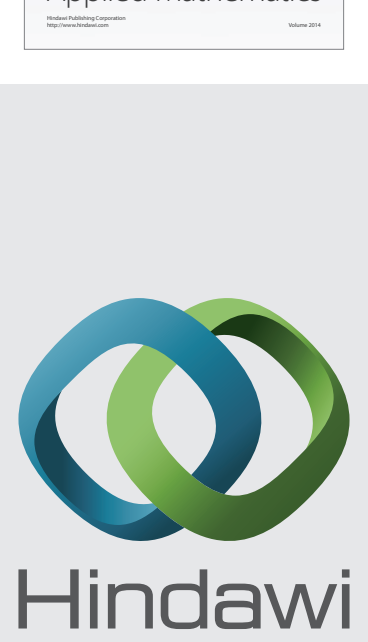

Submit your manuscripts at http://www.hindawi.com
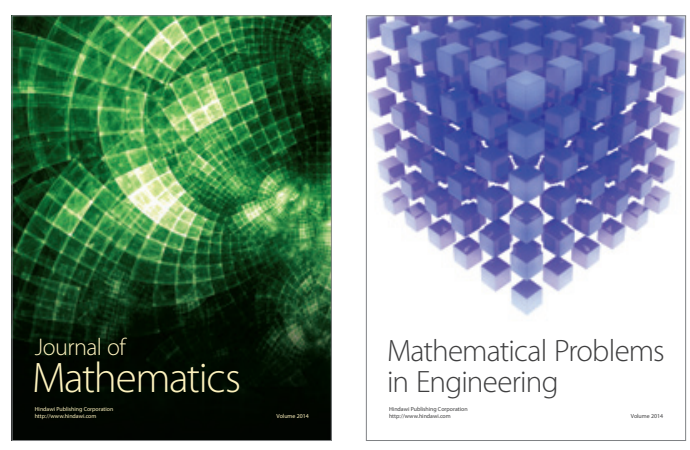

Mathematical Problems in Engineering
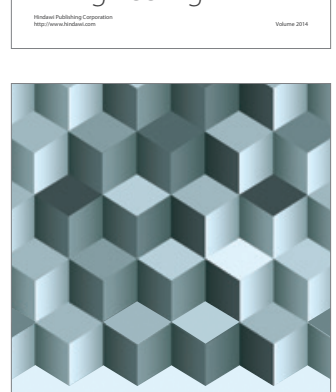

Journal of

Function Spaces
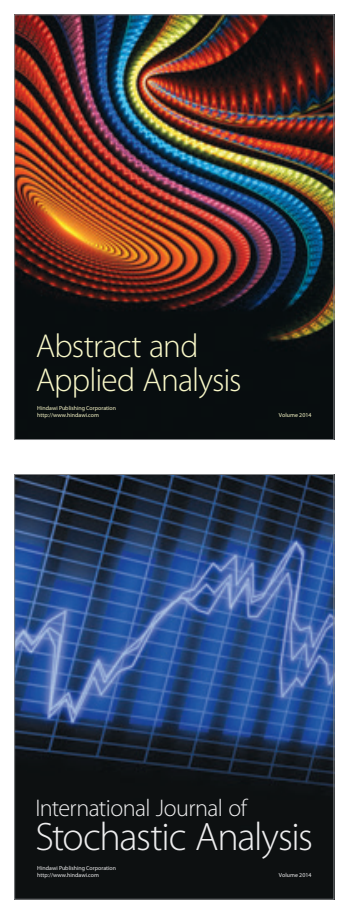

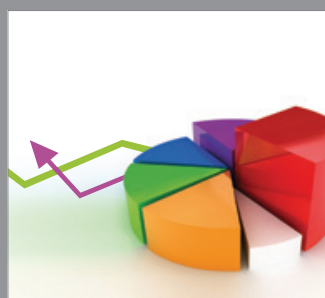

ournal of

Probability and Statistics

Promensencen
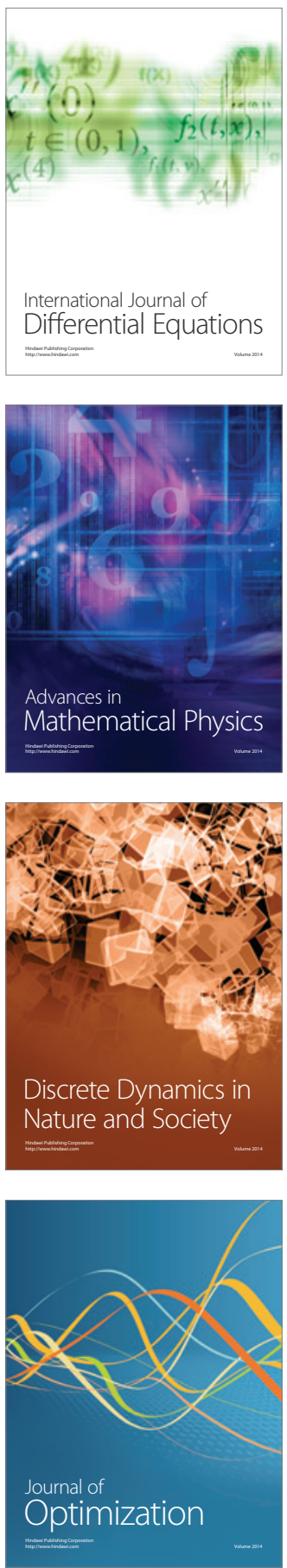